\title{
Determinación experimental del módulo de compresibilidad adiabático para aceites minerales
}

\author{
Experimental determination of adiabatic bulk modulus for mineral oils
}

\author{
William Prado Martínez, Mario Hoyos Mesa \\ Universidad Tecnológica de Pereira, Pereira, Colombia \\ pradodutp.edu.co, mhoyoseutp.edu.co
}

\begin{abstract}
Resumen- En este trabajo se propone un método y se diseña un equipo para la determinación experimental del módulo de compresibilidad adiabático como función de la presión y temperatura en aceites minerales utilizando ondas ultrasónicas. El módulo de compresibilidad adiabático se obtiene al multiplicar el cuadrado de la velocidad de propagación del sonido en el fluido por su densidad.
\end{abstract}

Palabras clave-Módulo de compresibilidad adiabático, compresibilidad, aceite mineral, velocidad del sonido en liquidos

\begin{abstract}
This paper proposes a method and an apparatus for the experimental determination of the adiabatic bulk modulus as a function of pressure and temperature in mineral oil using ultrasonic waves. The adiabatic bulk modulus is obtained by multiplying the square of the velocity of sound propagation in the fluid by density.
\end{abstract}

Key Word - Adiabatic bulk modulus, compressibility, mineral oil, speed sound in liquid

\section{INTRODUCCIÓN}

Todas las sustancias exhiben cambios de volumen por efecto de la presión, sin embargo, cuando estos cambios son mínimos suele asumirse que su comportamiento es incompresible. No obstante, esta asunción es inconveniente cuando el fluido se utiliza en sistemas hidráulicos que necesitan de una respuesta rápida, caso del control de las prensas de alta velocidad, los alerones en las aeronaves, tren de aterrizaje y timón de profundidad. Para obtener resultados coherentes con el comportamiento real, el estudio del comportamiento dinámico de estos sistemas exige que el fluido sea considerado como compresible.

La propiedad que tienen las sustancias de cambiar su volumen $V$ cuando son sometidas a cambios de presión se denomina compresibilidad. El inverso de la compresibilidad se denomina módulo de bulk $\beta$ que expresa la resistencia del fluido a la deformación volumétrica y está dado por la ecuación:

$$
\beta=-V \frac{\partial P}{\partial V}
$$

Un módulo de bulk bajo, indica que el fluido tiene un cambio considerable de volumen por efecto de una variación de la presión $P$. Para la misma variación de presión, un módulo de bulk alto indicará cambios mínimos en el volumen.

Los cambios de presión pueden realizarse a temperatura constante o entropía constante. Para el caso de procesos a temperatura constante el módulo de compresibilidad se denomina módulo de bulk isotérmico $\beta_{T}$ y está dado por la ecuación (2). Pueden encontrarse propuestas de medición del módulo isotérmico en Krawetz [7] y en Hoyos y Mejía [6].

$$
\beta_{T}=-\left(V \frac{\partial P}{\partial V}\right)_{T}
$$

Por otro lado, cuando los cambios de presión suceden sin que el sistema intercambie de calor con su entorno, el módulo de compresibilidad se denomina módulo de bulk adiabático $\beta_{S}$ y está dado por la expresión (3)

$$
\beta_{S}=-\left(V \frac{\partial P}{\partial V}\right)_{S}
$$

El módulo de bulk adiabático, se utiliza para procesos en los que no hay transferencia de calor hacia fuera o hacia adentro del sistema y se utiliza de forma aproximada cuando la transferencia de calor es mínima e insuficiente para mantener la temperatura constante. Esto es aplicable en sistemas donde los cambios en el volumen y/o presión del fluido suceden en intervalos de tiempo más pequeños que los necesarios para establecer procesos de transferencia de calor que alteren significativamente el comportamiento del sistema.

Por ejemplo, en condiciones de alta temperatura y presiones y elevadas velocidades, el módulo de bulk adiabático afecta significativamente el desempeño de los sistemas hidráulicos. 
Los tres efectos más relevantes son: La pérdida de energía por compresión, incremento de la constante de tiempo del sistema y picos de presión.

De acuerdo con [2], al aplicar alta presión a un volumen de líquido, una cantidad significativa de energía puede ser utilizada para comprimir el fluido, esencialmente apretando las moléculas del líquido entre sí. Esto reduce la eficiencia energética ya que el cambio de volumen del fluido no genera trabajo útil, aumenta significativamente los costos de operación y disminuye la autonomía de los equipos móviles. La Figura 1, muestra este efecto mediante la pérdida de potencia en un cilindro a 3000 psig por cada pulgada cúbica de fluido en función del tiempo para diferentes módulos de bulk.

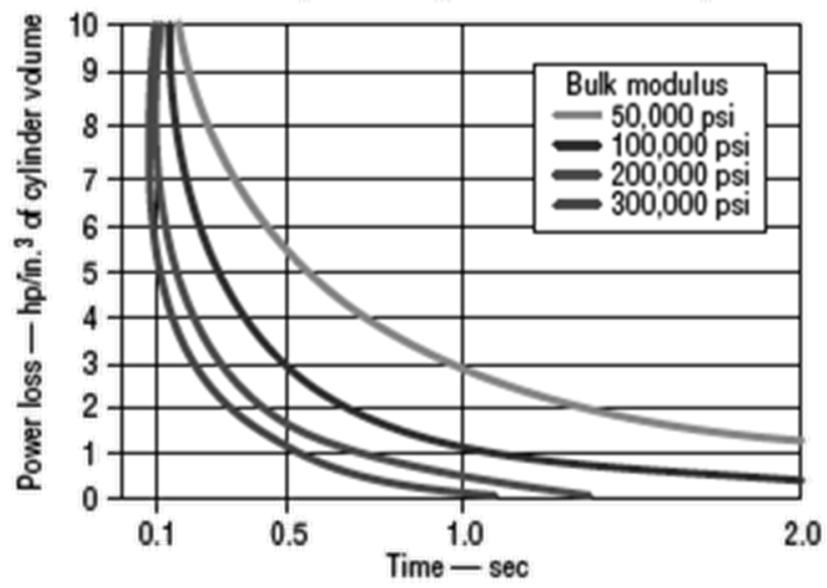

Figura 1. Pérdida de potencia en un cilindro a 3000 psig por cada in 3 como función del tiempo para diferentes módulos de bulk. Tomado de [2]

Los cambios de volumen del fluido, se traducen también en una respuesta tardía del sistema; el actuador puede no moverse hasta que el líquido aguas arriba ha sido comprimido y la energía almacenada en el líquido puede causar que el actuador siga avanzando después que la válvula de control se ha cerrado. Esto aumenta la constante de tiempo del sistema hidráulico, así como la complejidad del fenómeno para el acercamiento teórico.

Por otro lado, el módulo de bulk está relacionado con los picos de presión en un sistema hidráulico. Debido a efectos dinámicos como el cierre o apertura de válvulas y el inicio o parada de actuadores, se presentan transitorios y significativos aumentos de presión que pueden romper tuberías o inutilizar componentes. Cuanto mayor sea la rigidez del sistema y mayor sea el módulo de compresibilidad, mayor será el choque de presión que puede inutilizar los componentes hidráulicos y provocar fugas.

Para reducir los efectos perjudiciales, usualmente los sistemas hidráulicos contienen dispositivos que limitan las oscilaciones de presión. Sin embargo, esto puede no ser suficiente. Por ejemplo, para válvulas de alivio pequeñas el tiempo de apertura se encuentra entre 6 y 30 milisegundos, para válvulas más grandes el tiempo está entre 60 y 100 milisegundos. Durante este corto tiempo la bomba continúa descargando fluido y la presión del sistema se eleva porque los controles de presión no se anticipan para responder al flujo adicional que debe ser adsorbido por mangueras, válvulas, actuadores y otros componentes.

\section{MÉTODO DE MEDICIÓN}

Wood [11] y otros autores demuestran que la propagación del sonido en un recipiente o tubería cilíndricos satisface la ecuación de onda (4)

$$
\nabla^{2} \psi(\vec{r}, t)=\frac{1}{v^{2}} \frac{\partial^{2} \psi}{\partial^{2} t}(\vec{r}, t)
$$

Donde $v$ es la velocidad de propagación de la perturbación en el medio y $\vec{r}$ son coordenadas del espacio. Para ello proponen las siguientes asunciones:

- $\quad$ El sonido es una onda de presión longitudinal con velocidad de propagación en dirección al eje de la tubería

- $\quad$ Como las perturbaciones de presión son rápidas y la transmisión de calor es mínima, entonces es razonable suponer que tales perturbaciones son adiabáticas.

- $\quad$ Todas las perturbaciones son de pequeña amplitud y por lo tanto puede utilizarse la linealización de ecuaciones.

Para perturbaciones de presión la ecuación de onda (4), toma la forma de (5) donde $P$ es la presión y $\rho_{0}$ es la densidad en la dirección $x$

$$
\frac{\partial^{2} P}{\partial x^{2}}=\frac{\rho_{0}}{\beta_{S}} \frac{\partial^{2} P(x, t)}{\partial t^{2}}
$$

Comparando las ecuaciones (4) y (5) se deduce que el módulo de compresibilidad adiabático se obtiene al multiplicar el cuadrado de la velocidad de propagación de las ondas de presión en el fluido (velocidad del sonido) por su densidad.

$$
\beta_{S}=\rho_{o} v^{2}(6)
$$

La velocidad $v$ puede obtenerse dividiendo la distancia recorrida por la perturbación entre el tiempo empleado. Sin embargo, este método impone dos desafíos, para distancias cortas (centímetros) se necesitan órdenes de magnitud de nanosegundos para conseguir suficiente precisión necesaria en el análisis. Esto exige inversiones cuantiosas en instrumentación [5]. Si se aumenta el tiempo a un valor suficiente para que pueda ser registrado con los instrumentos 
tradicionales de laboratorio, se debe construir un recipiente del orden de metros. Esto introduce numerosas correcciones debido al comportamiento del material del recipiente $\mathrm{y}$ dificulta realizar pruebas a diferentes temperaturas.

La elección de utilizar la frecuencia como método para determinar la velocidad del sonido (perturbaciones), permite el uso de un recipiente rígido de pequeño volumen que puede incorporarse a un diseño como el de Mejía [9]. En el método se varía la frecuencia de una señal sinusoidal pura entregada a un emisor y se superpone con la señal recibida en unl receptor. La superposición es fácilmente observable en un osciloscopio mediante una figura de Lissajous o comparando los picos de las señales sinusoidales.

Para un comportamiento sinusoidal la señal de presión toma la forma

$$
P(x, t)=P_{0} \operatorname{Sin}(k x-2 \pi f t)
$$

Si una señal se emite en $x=0$ y es observada a una distancia $x=L$, la ecuación (7) permite obtener expresiones para la señal emitida $\psi_{E}(0, t)$ y la observada o captada $\psi_{R}(L, t)$ transformándose en (8) y (9)

$$
\begin{gathered}
\psi_{E}=\psi_{0 E} \operatorname{Sin}(-2 \pi f t), x=0 \\
\psi_{R}=\psi_{0 R} \operatorname{Sin}(k L-2 \pi f t), x=L
\end{gathered}
$$

Las ecuaciones (8) y (9) pueden escribirse como

$$
\begin{gathered}
\operatorname{Sin}(-2 \pi f t)=\frac{\psi_{E}}{\psi_{0 E}} \\
\operatorname{Cos}(-2 \pi f t)=\frac{1}{\operatorname{Sin}(k L)}\left(\frac{\psi_{R}}{\psi_{0 R}}-\frac{\psi_{E}}{\psi_{0 E}} \operatorname{Cos}(k L)\right)
\end{gathered}
$$

De acuerdo con Wood [11], es posible combinar las ecuaciones (10) y (11) como dos vibraciones armónicas simples mutuamente perpendiculares eliminando el parámetro $t$ de ambas ecuaciones. Elevando al cuadrado y sumando se obtiene:

$$
\frac{\psi_{R}^{2}}{\psi_{0 R}^{2}}-\frac{2 \psi_{R} \psi_{E}}{\psi_{0 R} \psi_{0 E}} \operatorname{Cos}(k L)+\frac{\psi_{E}^{2}}{\psi_{0 E}^{2}}-\operatorname{Sin}^{2}(k L)=0
$$

En general la ecuación (12) describe una elipse inscrita en el rectángulo $\psi_{E}= \pm \psi_{0 E}, \quad \psi_{R}= \pm \psi_{0 R}$ como muestra la figura 2 .

Cuando la figura de Lissajous degenera a una línea recta, los picos de las señales sinusoidales se encuentran en fase. La frecuencia para la cual la señal del emisor y la señal del receptor entran en fase se multiplica por la distancia de separación entre los transductores para obtener la velocidad de la perturbación en el fluido.

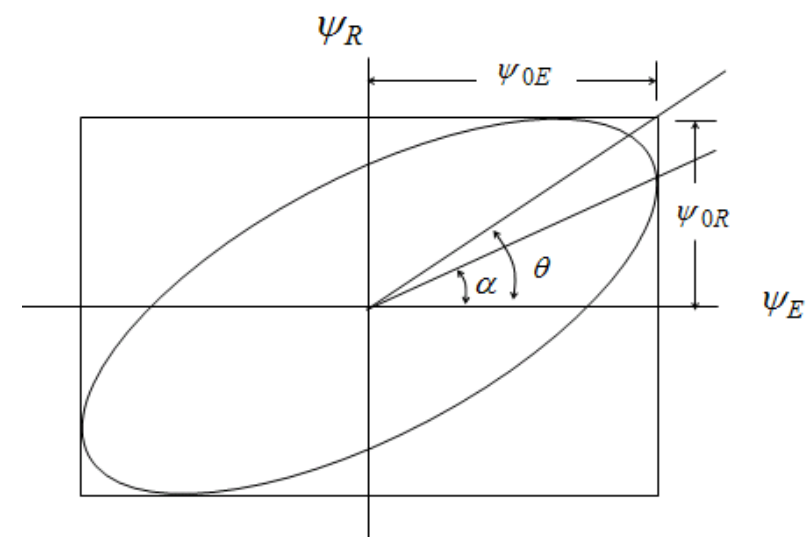

Figura 1. Figura de Lissajous

De la figura 2 puede deducirse que

$$
\begin{gathered}
\operatorname{Tan} \theta=\frac{\psi_{0 R}}{\psi_{0 E}} \\
\operatorname{Tan} \alpha=\operatorname{Cos}(k L) \operatorname{Tan} \theta
\end{gathered}
$$

La figura 3 muestra los modos de vibración para $\lambda=L$ y $\lambda=L / 2$

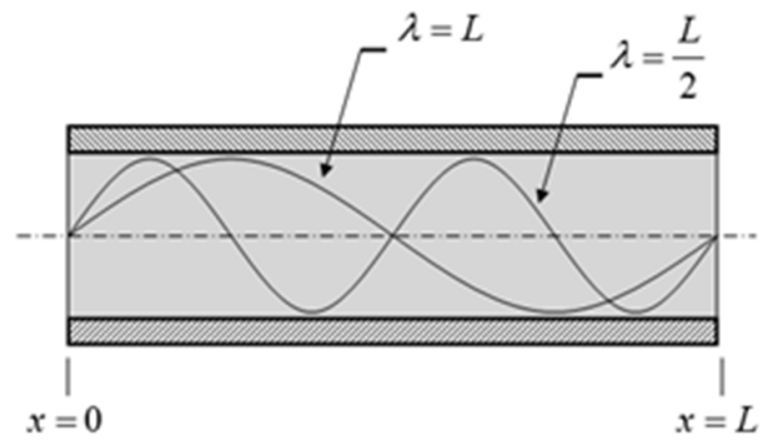

Fig 3. Modos de vibración

En general si $\lambda=L / n$ con $n=1,2,3 \ldots$ el producto $k L=2 \pi n$ causando que la señal emitida $\psi_{E}(0, t)$ y la observada o captada $\psi_{R}(L, t)$ estén en fase y por lo tanto la elipse de la ecuación (12) degenera en una línea recta dada por

$$
\psi_{R}=\frac{\psi_{0 R}}{\psi_{0 E}} \psi_{E}
$$

Variando la frecuencia $f$ puede conseguirse que la longitud de onda alcance modos de vibración dados por $\lambda=L / n$ con

$n=1,2,3 \ldots$, en este caso la velocidad del sonido estará dada por

$$
v=\frac{L}{n} f
$$

\section{SISTEMA DE medición}

El equipo cuenta con un recipiente que contiene aceite hidráulico a presión. Dentro del recipiente e inmerso en el 
aceite se encuentran un emisor y un receptor ultrasónico. El proceso se visualiza superponiendo las señales del emisor y del receptor para formar una figura de Lissajous en el osciloscopio.

Como la velocidad de propagación cambia con la presión y la temperatura, se realiza un diseño experimental con variables presión y temperatura. Los estímulos de presión se controlan mediante una bomba de pistón y para controlar la temperatura se sumerge el recipiente en un baño termostático. El sistema de medición desarrollado se muestra en la figura 4 .

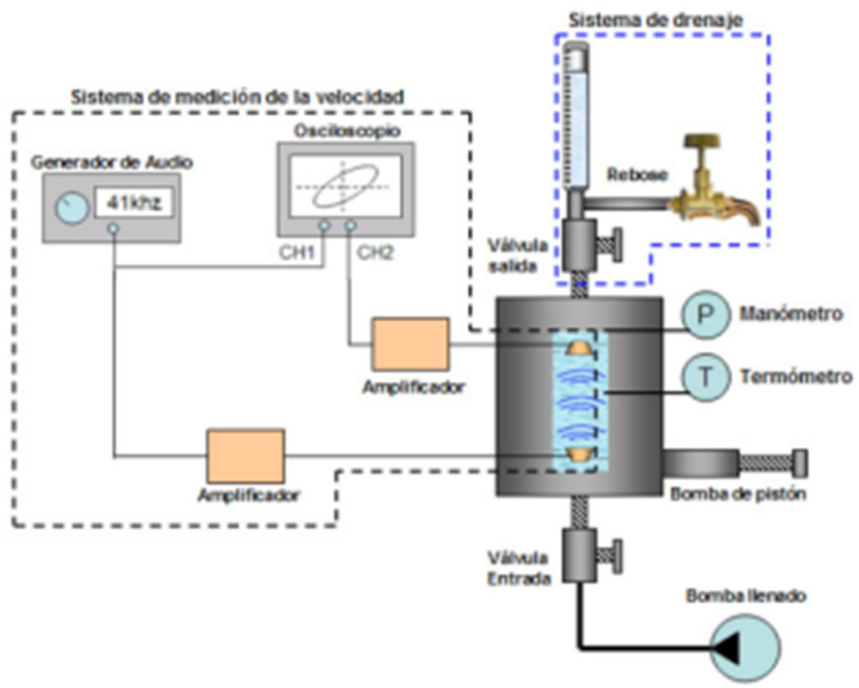

Figura 4. Sistema de medición para el módulo de compresibilidad adiabático

El rango de presiones utilizado es de 0 psi a 5000 psi manométricas y se utilizan dos temperaturas diferentes $25^{\circ} \mathrm{C} \mathrm{y}$ $60^{\circ} \mathrm{C}$. La densidad se calcula mediante el cambio de volumen del aceite dado por el avance del tornillo de la bomba de pistón cuando las válvulas de entrada y salida están cerradas.

La bomba de pistón puede utilizarse para corregir la densidad $\rho$ del fluido como función del avance $x_{z}$ del tornillo en milímetros mediante la ecuación (7), donde $\rho_{0}$ es la densidad inicial del fluido, $A_{p}$ el área de sección transversal del pistón y $V_{0}$ el volumen inicial del recipiente.

$$
\rho=\frac{\rho_{0}}{1-\frac{A_{p} x_{z}}{V_{0}}}
$$

La longitud entre transductores puede cambiar debido a que los materiales de los que están construidos cambian sus dimensiones bajo los efectos de la presión y de la temperatura. El cambio en las dimensiones de los transductores altera la distancia L entre estos y por lo tanto la medición del módulo de compresibilidad.
Para corregir estos efectos se elabora un modelo matemático que predice los cambios de longitud entre transductores como una función de la presión y de la temperatura. Dado que en general, los cambios en las dimensiones de los materiales son lineales con la temperatura y la presión, el modelo que se emplea es un modelo multilineal, para ello se hace uso de la herramienta Solver de Microsoft Excel ${ }^{\circledR}$. La distancia inicial L inicial entre transductores se establece utilizando un calibrador. La ecuación de corrección para la distancia entre sensor y emisor como función de la presión y de la temperatura es:

$$
L_{P, T}=36,73-0,00105(T-25)+0,03677 P
$$

Con la longitud $L_{P, T}$ en milímetros, la temperatura $\mathrm{T}$ en grados centígrados y la presión $\mathrm{P}$ en $\mathrm{MPa}$. La velocidad $v$ está dada entonces por la ecuación (9) donde $f$ es la frecuencia

$$
v=f L_{P, T}
$$

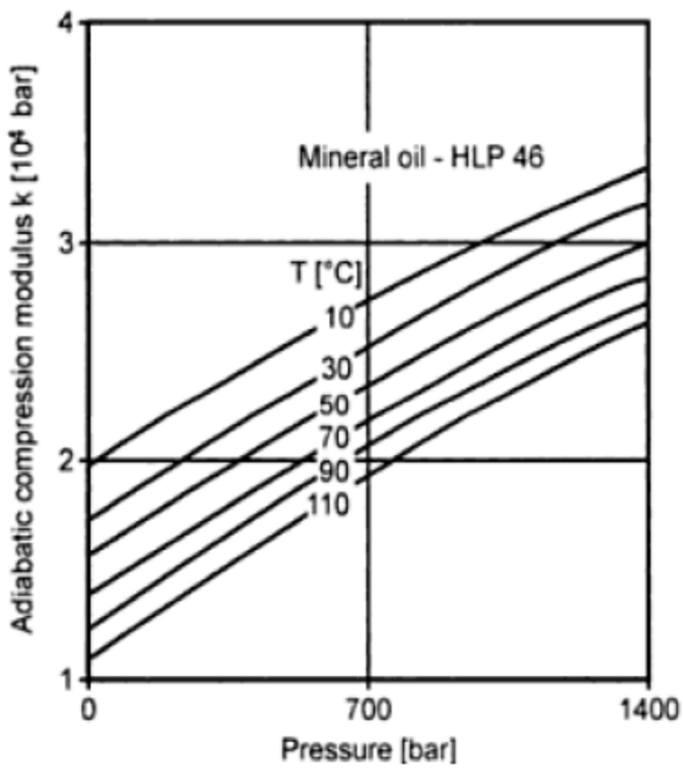

Figura 5. Módulo de compresibilidad adiabático

La incertidumbre en la medición del módulo de compresibilidad con el aparato se determina con la ecuación

$$
\Delta \beta_{A}=v^{2} \Delta \rho+2 v \rho \Delta v
$$

Donde $\Delta \rho$ y $\Delta v$ son los errores considerando las incertidumbres de medición del equipo y los errores estadísticos

\section{RESULTADOS}


Para aceite Shell Tellus 46 con $\Delta \rho= \pm 1,72 \mathrm{~kg} / \mathrm{m}^{3}, \Delta v$ $= \pm 6,95 \mathrm{~m} / \mathrm{s}, \quad v=1555 \mathrm{~m} / \mathrm{s}, \rho=896 \mathrm{~kg} / \mathrm{m}^{3}$ se obtiene que la máxima incertidumbre del equipo es $\Delta \beta_{A}= \pm 0,03 \mathrm{GPa}$.

Considerando que para los valores anteriores $\beta_{A}=2,17 \mathrm{GPa}$, entonces $\Delta \beta_{A}= \pm 1,4 \%$ del valor de $\beta_{A}$.

La tabla 1 muestra el resumen de los valores obtenidos para la medición del módulo de compresibilidad adiabático a $25^{\circ} \mathrm{C}$ y su error para cada valor de presión.

Tabla 1. Módulo de bulk como función de la presión a $25^{\circ} \mathrm{C}$

\begin{tabular}{|c|c|c|c|c|c|c|}
\hline Temperatura 25 ${ }^{\circ} \mathrm{C}$ & \multicolumn{5}{|c|}{ Tabla del módulo de compresibilidad (GPa) } \\
\hline Presión psig & 0 & 1000 & 2000 & 3000 & 4000 & 5000 \\
\hline Presión MPa & 0,00 & 6,89 & 13,79 & 20,68 & 27,57 & 34,46 \\
\hline bulk Medido 25 \% C & 1,80 & 1,86 & 1,94 & 2,02 & 2,10 & 2,17 \\
\hline bulk Referencia 25 ${ }^{\circ} \mathrm{C}$ & 1,80 & 1,87 & 1,95 & 2,03 & 2,10 & 2,19 \\
\hline Discrepancia \% & $0,00 \%$ & $0,43 \%$ & $0,48 \%$ & $0,35 \%$ & $0,22 \%$ & $1,03 \%$ \\
\hline
\end{tabular}

De las mediciones obtenidas para el módulo de compresibilidad a $25^{\circ} \mathrm{C}$, se tiene que la máxima diferencia respecto a los valores publicados en la literatura (figura 5) se presenta para un valor de presión de $34,56 \mathrm{MPa}$ (5000psig). Para este punto, el valor del módulo medido es de $(2,17 \pm 0,02) \mathrm{GPa}$ y el valor publicado por Mang[8] es $2,19 \mathrm{GPa}$, la diferencia o discrepancia en este punto es de 1,03\% (Tabla 1). Aún con las diferencias, las mediciones realizadas con el aparato se encuentran bajo control estadístico. La figura 6 muestra un comportamiento lineal del módulo de compresibilidad como función de la presión a $25^{\circ} \mathrm{C}$ con respecto a los valores de referencia.

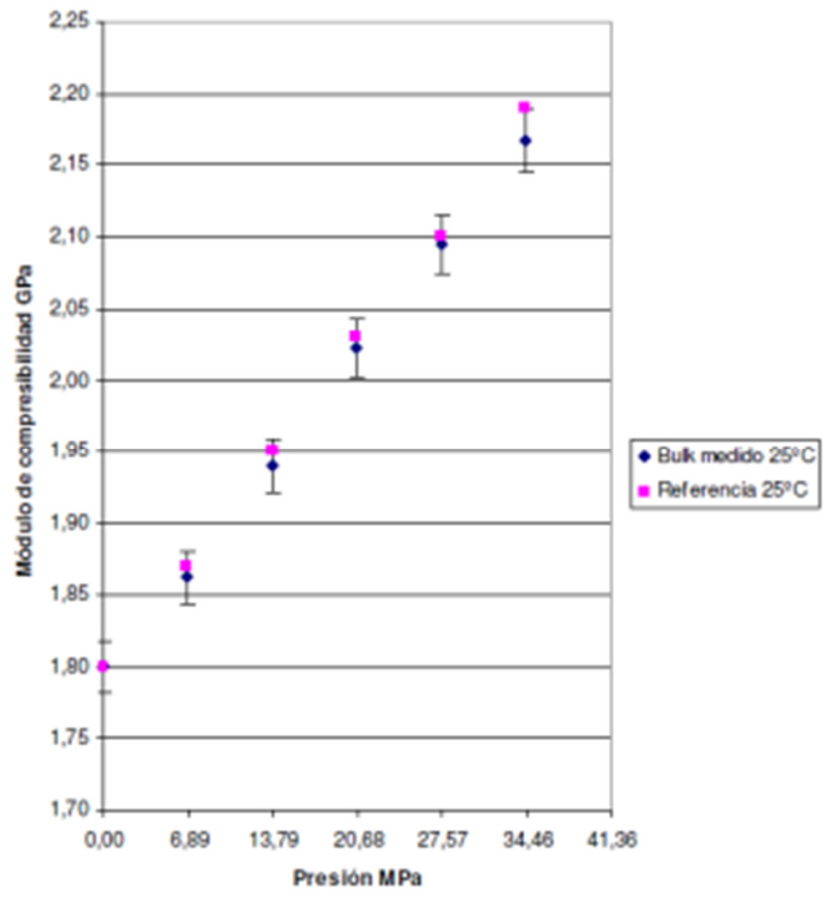

Figura 6. Módulo de compresibilidad adiabático a $25^{\circ} \mathrm{C}$

Para el módulo de compresibilidad a $60^{\circ} \mathrm{C}$, la máxima diferencia respecto a los valores publicados por Mang[8] se presenta también para la máxima presión del experimento 34,56MPa (5000psig). En este punto, el valor del módulo medido es de $(1,89 \pm 0,02) \mathrm{MPa}$ y el valor publicado es $1,86 \mathrm{GPa}$, la diferencia o discrepancia para este punto es de $1,25 \%$. La tabla 2 muestra en resumen los valores obtenidos para la medición del módulo de compresibilidad adiabático y su error para cada tratamiento a $60^{\circ} \mathrm{C}$.

Tabla 2. Módulo de bulk como función de la presión a $60^{\circ} \mathrm{C}$

\begin{tabular}{|c|c|c|c|c|c|c|}
\hline Temperatura $60^{\circ} \mathrm{C}$ & \multicolumn{5}{|c|}{ Tabla del modulo de compresibilidad (GPa) } \\
\hline Presión psig & 0 & 1000 & 2000 & 3000 & 4000 & 5000 \\
\hline Presion MPa & 0,00 & 6,89 & 13,79 & 20,68 & 27,57 & 34,46 \\
\hline bulk Medido $600^{\circ} \mathrm{C}$ & 1,45 & 1,55 & 1,64 & 1,73 & 1,81 & 1,89 \\
\hline bulk Referencia $60^{\circ} \mathrm{C}$ & 1,47 & 1,56 & 1,64 & 1,72 & 1,80 & 1,86 \\
\hline Discrepancia \% & $1,34 \%$ & $0,91 \%$ & $0,06 \%$ & $0,41 \%$ & $0,51 \%$ & $1,25 \%$ \\
\hline
\end{tabular}

La figura 7 muestra el comportamiento del módulo como función de la presión a $60^{\circ} \mathrm{C}$ con respecto a los valores de referencia. En ambos casos el comportamiento es lineal.

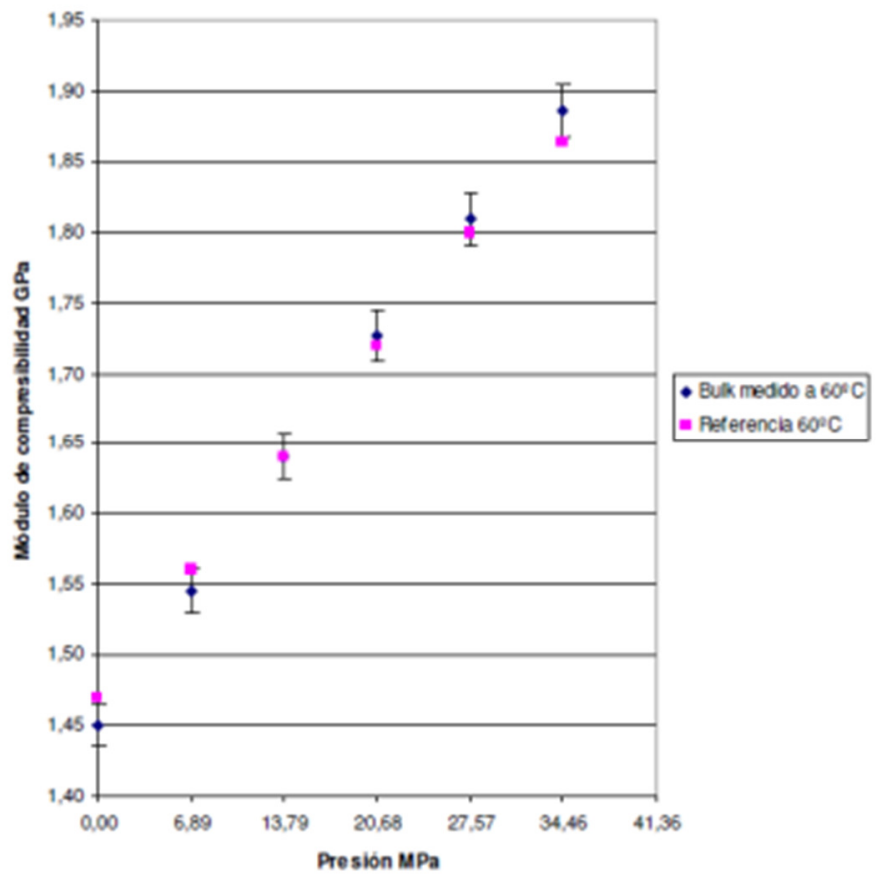

Figura 7. Módulo de compresibilidad adiabático a $60^{\circ} \mathrm{C}$

Con la presión en MPa y el módulo de compresibilidad en $\mathrm{GPa}$, las regresiones lineales para el módulo de compresibilidad son: Para una temperatura de $25^{\circ} \mathrm{C}$, $\beta_{A}=0,0109 P+1,743$ y coeficiente de correlación $r^{2}=0,9988$.Para una temperatura de $60^{\circ} \mathrm{C}$, $\beta_{A}=0,0127 P+1,74583$ y coeficiente de correlación. 
La figura 8 muestra el módulo de bulk como función de la presión a $25^{\circ} \mathrm{C}$ y $60^{\circ} \mathrm{C}$. Las dos rectas interpretan el comportamiento del módulo con la presión para más del $99 \%$ de las mediciones.

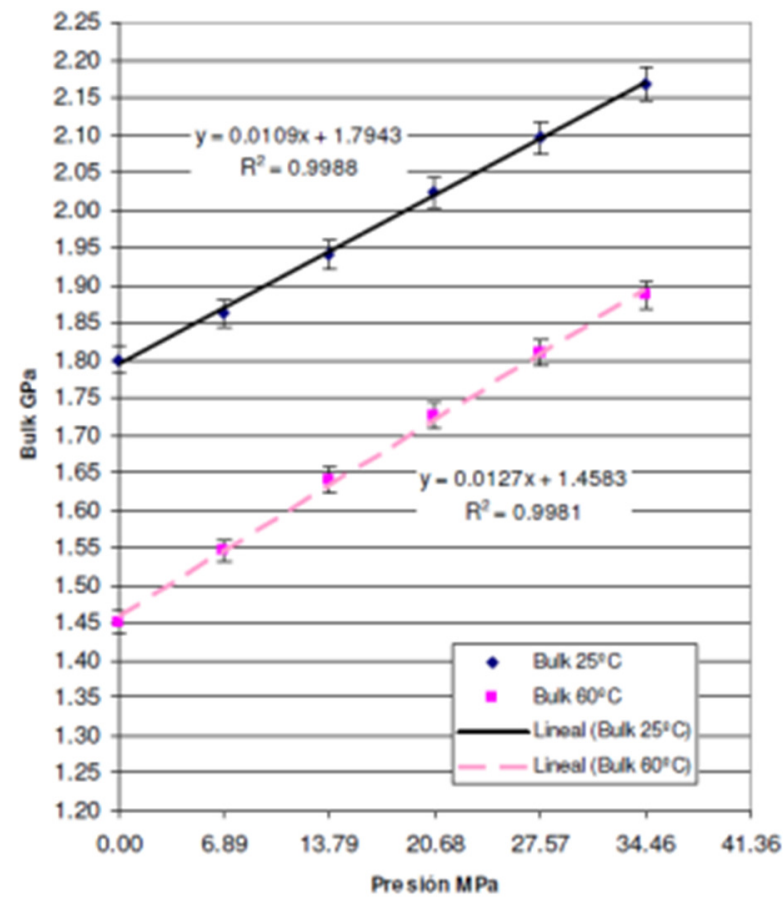

Figura 8. Módulo de bulk adiabático a $25^{\circ} \mathrm{C}$ y $60^{\circ} \mathrm{C}$

\section{CONCLUSIONES Y RECOMENDACIONES}

Se desarrolló un equipo de medición para el módulo de compresibilidad adiabático mediante ultrasonido y se propuso un método mediante la composición de ondas mutuamente perpendiculares y figuras de Lissajous. El equipo desarrollado presenta una incertidumbre del $1,25 \%$ de acuerdo con el análisis realizado. El mayor aporte a la incertidumbre se debe a la medición de la velocidad del sonido, mientras el menor aporte se debe a la medición de la densidad. El valor calculado considera los cambios en la longitud entre transductores debidos a la presión entre 0 -5000psig y la temperatura entre $20^{\circ} \mathrm{C}-60^{\circ} \mathrm{C}$.

Cualquier cambio en el montaje de los transductores requiere de una nueva calibración del aparato. Como el comportamiento de la longitud con la presión y la temperatura es lineal, solo bastan cuatro puntos para realizar la calibración y determinar el modelo matemático para corregirla. Los puntos necesarios son: dos puntos a presión atmosférica y temperatura diferente, los otros dos puntos para la máxima presión (5000 psig) con las mismas temperaturas. Dadas las especificaciones de los transductores no se recomienda su uso para temperaturas mayores a $80^{\circ} \mathrm{C}$.
Se constató un comportamiento similar del módulo de compresibilidad del aceite Shell Tellus 46 con respecto a lo publicado por Mang[8]. La máxima diferencia entre los valores fue de $1.25 \%$. Es de anotar que en la publicación mencionada no se muestra la incertidumbre de los datos.

Para las mediciones realizadas se encontró que los resultados se encuentran bajo control estadístico. Esto quiere decir: primero, que existen relaciones entre la temperatura y la presión con los valores obtenidos para el módulo de compresibilidad y segundo que estas relaciones se pueden explicar con las ecuaciones obtenidas. Por otro lado, las mediciones realizadas para una prueba del $95 \%$ de nivel de confianza reportaron un valor máximo del 3.8\%, lo cual quiere decir, que se puede confiar de los resultados en un $96.2 \%$.

Con el equipo y el método propuestos se consiguió una incertidumbre del $10 \%$, es decir menor a la propuesta. Esto permite que el sistema pueda ser utilizado para realizar la caracterización de otros aceites hidráulicos.

Adicionalmente, se sugiere implementar el equipo para la medición de otras sustancias, en especial de biodiesel por la importancia actual de este combustible en el parque automotor nacional. Un paso necesario para este cometido es identificar la compatibilidad de los transductores con otras sustancias.

\section{REFERENCIAS}

[1] Balasubramanian, Karthik. "Smart bulk modulus sensor". A thesis presented to the graduate school of the University of Florida in partial fulfillment of the requirements for the degree of Master of Science, university of Florida 2003, pp. 5,11

[2] Barber, Allen; George, Herman H. "What is bulk modulus, and when is it Important?" Hydraulics \& Pneumatics Date: July 1, 2007

[3] Blackburn J.F, Reethof G., Shearer J.L. "Fluid Power Control". The Massachusetts Institute and Technology, Cambridge, 1960.

[4] Corbo, Mark A. Stearns Charles. "Practical desing against pumps pulsations". Proceedings of the twenty-second international pumps users simposium. 2005

[5] Hoyos Mesa, Mario. Mejía, Luz Adriana. Henao, Edison. "Determinación experimental del módulo de bulk de tuberías flexibles para sistemas oleohidráulicos" Scientia et técnica, Año XI - Número 29, pp. 151-156, Dic 2005

[6] Hoyos Mesa, Mario, Mejía Luz Adriana, "Sistema para medir la compresibilidad isotérmica de aceites minerales" Scientia et técnica, Año XII - Número 32, pp. 213-216, Dic 2006

[7] Krawetz, Arthur A. "Method for the measurement bulk modulus and pressure viscosity of liquids", U.S. Patent 5 383 352, Jun. 4, 1995 
[8] Mang, Theo. Dresel, Wilfried. "Lubricants and lubrication". Second edition. Wiley- VCH, pp. 308-331. 2007

[9] Mejía, Luz Adriana. "Construcción de un sistema para medir la compresibilidad isotérmica de aceites minerales". Tesis de Maestría en Sistemas Automáticos de Producción. Universidad Tecnológica de Pereira. 2005.

[10] Surawattanawan, Prakob. "An Approach for the Identification of Hydraulic Oil Bulk Modulus Utilizing Wave Propagation Effect and FFT". The 17 Conference of mechanical engineering network of Thailand. Oct 2003.

[11] Wood, Alexander. "Acoustics". Dover Publications, Inc. New York 1966, pp 208 